Service social

\title{
Les abus sexuels envers les enfants. Jalons dans la formation d'un partenariat
}

\author{
John Wright, Stéphane Sabourin, Thomas Lebeau, Jacqueline \\ Oxman-Martinez, Alain Perron et Yvan Lussier
}

Volume 43, numéro 2, 1994

Enfants, parents, intervenants

URI : https://id.erudit.org/iderudit/706654ar

DOI : https://doi.org/10.7202/706654ar

Aller au sommaire du numéro

Éditeur(s)

École de service social de l'Université Laval

ISSN

1708-1734 (numérique)

Découvrir la revue

Citer cet article

Wright, J., Sabourin, S., Lebeau, T., Oxman-Martinez, J., Perron, A. \& Lussier, Y. (1994). Les abus sexuels envers les enfants. Jalons dans la formation d'un partenariat. Service social, 43(2), 7-29. https://doi.org/10.7202/706654ar
Résumé de l'article

L'objectif de cet article consiste à exposer les résultats préliminaires d'un processus de consultation et de concertation entre cinq Centres jeunesse et cinq chercheurs, au moment de la création d'un partenariat autour de la problématique de l'abus sexuel à l'endroit des enfants. Après avoir rappelé diverses statistiques sur la prévalence du phénomène, les auteurs présentent les résultats d'une recension des écrits sur le profil psychosocial des différents acteurs dans l'abus sexuel (la victime, le ou les parents non abuseurs, l'abuseur et la famille) et sur la réponse institutionnelle, telle que : le choix des mesures, le placement de l'enfant ainsi que les multiples formes de thérapie. Ils discutent des priorités des recherches descriptive et évaluative, de même que des multiples défis à surmonter afin de mener des recherches d'une rigueur scientifique qui auront des retombées immédiates pour les intervenants dans ce domaine de pointe. 


\section{$A R T I C L: S$}

\section{Les abus sexuels envers les enfants}

Jalons dans la formation d'un partenariat'

John WRIGHT ${ }^{2}$ Département de psychologie Université de Montréal

Stéphane SABOURIN

Université Laval

Thomas LEBEAU

Centres jeunesse de Lanaudière Jacqueline OXMAN-MARTINEZ Centres jeunesse de la Montérégie

Alain PERRON Centres jeunesse du Centre-du-Québec

Yvan LUSSIER

Université du Québec à Trois-Rivières

1. Cette recherche a été rendue possible grâce à une subvention de recherche octroyée au Partenariat de recherche et d'intervention en matière d'abus sexuel (PRIMASE) par le Conseil québécois de la recherche sociale (CQRS).

2. Les personnes suivantes ont également apporté une contribution appréciable à cet article: Francine Sénécal-Brooks, Louise Mercure, Claude Bélanger, Jean-Pierre Paradis, Guy Moffatt et Michel Tremblay. 
L'objectif du présent article est d'exposer les résultats préliminaires $d^{\prime}$ 'un processus de consultation et de concertation entre des représentants de cinq Centres de protection de l'enfance et de la jeunesse (CPEJ) et cinq chercheurs, au moment de la création d'un partenariat (PRIMASE) autour de la problématique de l'évaluation et du traitement de l'abus sexuel d'enfant. Ce partenariat regroupe des chercheurs rattachés à I'Université de Montréal, à l'Université Laval, à l'Université du Québec à Trois-Rivières, aux Centres jeunesse de la Montérégie et à l'hôpital Douglas, de même que des intervenants issus des Centres jeunesse du Centre-du-Québec, Saguenay - Lac-Saint-Jean, Estrie, LaurentidesLanaudière et Montérégie. Les membres du partenariat poursuivent trois objectifs. Premièrement, nous nous proposons d'examiner le profil psychosocial des différents acteurs dans I'abus sexuel intrafamilial et extrafamilial. Deuxièmement, nous comptons analyser l'efficacité de la réponse institutionnelle en matière $d$ 'abus sexuel. Troisièmement, nous désirons mettre sur pied une seconde génération d'études guidées par les consultations menées dans les Centres jeunesse et par les résultats des premières recherches descriptives et évaluatives entreprises. Plus spécifiquement, il s'agira de:

1) déterminer les répercussions à long terme des services offerts dans les Centres jeunesse en matière d'abus sexuel: diminution, stabilisation ou accroissement des séquelles psychosociales, revictimisation, éclatement de la famille et définition des facteurs, judiciaires, familiaux et thérapeutiques, associés à l'amélioration de la situation ;

2) évaluer les besoins de formation et les mesures institutionnelles de soutien à améliorer afin de contrer la mobilité élevée, dans certains Centres jeunesse, des intervenants qui œuvrent dans ce secteur ;

3) développer et évaluer les modèles décisionnels utilisés par les différents acteurs concernés (mère, intervenant, système juridique), afin de déterminer la nature et la fréquence des contacts entre le père abuseur et la victime et ;

4) mener des recherches descriptives qui visent à cerner I'ampleur et les répercussions des formes d'abus sexuel apparemment en croissance: I'abus sexuel par d'autres jeunes, l'abus sexuel en famille d'accueil et l'abus sexuel en centre de réadaptation pour jeunes en difficultés d'adaptation (CRJDA).

L'atteinte de ces objectifs nécessite la mise en place de divers processus de consultation de la documentation scientifique et clinique traitant de l'abus sexuel. Dans le cadre du présent article, après avoir rappelé diverses statistiques sur la prévalence du phénomène, nous présentons les résultats d'une recension des écrits sur le profil 
psychosocial des différents acteurs dans I'abus sexuel. Dans un second article (Wright, Sabourin, Lussier et Bélanger, 1995), nous recensons la littérature descriptive et évaluative qui porte sur la réponse institutionnelle, telle que : le choix des mesures, le placement de l'enfant, les multiples formes de thérapie, etc. C'est à partir de cet ensemble de données préliminaires que diverses consultations sont effectuées pour déterminer les priorités parmi les activités à entreprendre dans le but de solidifier le partenariat: enquêtes internes, analyse des besoins, rencontres avec les intervenants et les administrateurs de programmes, examen des défis organisationnels, etc.

L'analyse des recherches étiopathogéniques recensées s'appuie sur le postulat selon lequel I'adoption d'une approche descriptive systématique $^{3}$ permettra de mieux rendre compte:

1) de la nature, de l'ampleur, de l'intensité et de la chronicité des séquelles psychosociales observées chez les victimes d'abus sexuel (p. ex. troubles affectifs, difficultés d'apprentissage, stress posttraumatique, troubles somatiques, comportements sexualisés, problèmes de comportement, troubles anxieux, troubles dissociatifs, enfants asymptomatiques, etc.) ;

2) de la diversité des caractéristiques sociodémographiques et des troubles psychosociaux notés chez les abuseurs (p. ex. troubles psychopathiques, faible capacité d'empathie, style d'attachement déficient ou érotisé, dynamique de désinvestissement du père, excitation sexuelle déviante, etc.) et;

3) de la multiplicité des structures et des modes d'organisation des relations au sein des familles des victimes $d$ 'abus sexuel (p. ex. structures chaotiques ou à l'inverse hyper-rigides; faible différenciation des structures identitaires; inversion des rôles mèrefille; répartition inégale du pouvoir: dominance matriarcale ou patriarcale, etc.).

\section{ÉTAT DE LA SITUATION}

\section{La définition de l'abus et l'incidence}

La définition de l'abus sexuel retenue par la Table des directeurs de la protection de la jeunesse (1991) est la suivante: Geste posé par une personne donnant ou recherchant une stimulation sexuelle non

3. Ici, nous préconisons le terme «systématique » et nous ne faisons nullement référence à l'école "systémique». 
appropriée quant à l'âge et au niveau de développement de l'enfant ou de l'adolescent-e, portant ainsi atteinte à son intégrité corporelle ou psychique, alors que l'abuseur a un lien de consanguinité avec la victime ou qu'il est en position de responsabilité, d'autorité ou de domination avec elle. Plusieurs débats portent sur les diverses dimensions de cette définition. Par exemple, certains auteurs (Larouche, 1994) estiment que le terme "agression sexuelle», au lieu de "abus sexuel», reflète mieux la nature des gestes et les motivations de l'abuseur. En outre, plusieurs membres de l'actuel partenariat s'interrogent sur les risques que pourrait amener l'application de l'actuelle définition quant au lien entre la victime et l'agresseur, à savoir l'exclusion de plusieurs types d'abus par des tiers majeurs ou mineurs. La définition du terme "abus sexuel» a un impact direct sur de multiples thématiques, telles les statistiques sur la prévalence et l'incidence.

Les statistiques canadiennes (Badgley et al., 1984 ; Bagley et Ramsey, 1986) et américaines (Finkelhor, 1994) sur la prévalence montrent qu'avant l'âge de 18 ans approximativement une femme sur trois et un homme sur six seront victimes d'abus sexuels intra ou extrafamiliaux (voir aussi le rapport produit par Tourigny, 1991). En ce qui concerne le nombre de cas relevés par les Centres jeunesse (taux d'incidence), il faut souligner une progression constante du nombre de cas d'abus sexuels signalés. Au Québec, en cinq ans, la proportion de cas d'abus sexuels pris en charge a presque doublé (CPDJ, 1990), tandis qu'au Canada le taux a quintuplé en trois ans (Badgley et al., 1984); aux ÉtatsUnis, dans certains États, le nombre de cas signalés a presque décuplé sur une période de dix ans (Eckenrode, Powers, Doris, Munsch et Bolger, 1988; Finkelhor, 1994).

Fait à noter, tant au Québec qu'aux États-Unis, la croissance des signalements d'abus sexuel est apparemment plus forte que la progression des autres formes de mauvais traitements. De plus, des chercheurs américains ont noté que le nombre de signalements d'abus sexuel corroborés est plus élevé que le nombre de signalements pour les autres formes de mauvais traitements (Eckenrode et al., 1988). Malheureusement, ces statistiques ne permettent pas toujours de distinguer l'abus sexuel intra et extrafamilial, de telle sorte qu'on ne peut pas savoir si ces deux formes d'abus évoluent à la même vitesse.

Cependant, plusieurs variations dans les méthodes de calcul du taux annuel d'abus sexuels suivis en Centres jeunesse soulignent la nécessité d'interpréter avec prudence toutes les statistiques sur I'incidence. Premièrement, il faut préciser si la statistique renvoie au taux de cas rapportés ("signalés»). Les équipes en "Réception et traitement des signalements »(RTS) ont pour tâche de décider s'il y a 
suffisamment de matière pour procéder à une évaluation plus approfondie. L'évaluation des données descriptives des cinq Centres jeunesse membres du PRIMASE révèle une importante variation dans le taux de rétention des signalements à la fois selon les CPEJ et selon les années (Wright et al., 1994). Deuxièmement, il faut savoir quel pourcentage des signalements est jugé fondé («faits fondés » = FF) par l'équipe $\mathrm{d}^{\prime}$ «Évaluation et Orientation» (É-O). Le taux de FF se situe autour de $75 \%$ dans les cinq CPEJ participants; toutefois, ce taux varie beaucoup entre les Centres jeunesse et d'une année à l'autre. L'équipe É-O doit aussi décider si la sécurité et le développement de l'enfant sont compromis (SDC). De façon générale, dans les Centres jeunesse, on a tendance à décider que la sécurité et le développement sont compromis dans les cas d'abus réels par le père biologique ou le conjoint de fait, et à juger la sécurité et le développement non compromis dans les cas d'abus par un tiers - dans la mesure où la capacité parentale est jugée adéquate. Cependant, puisqu'il n'y a pas encore de système normalisé pour la tenue de statistiques sur les taux de SDC en fonction du lien entre la victime et l'abuseur, ou pour la pondération des facteurs de risque dans les cas d'abus intrafamilial comparativement à extrafamilial, il n'est pas surprenant de constater une variation des taux de SDC selon les Centres jeunesse et selon les années. Finalement, la fréquence $d^{\prime}$ 'abus sexuels peut renvoyer au taux d'application des mesures par les Centres jeunesse. À la suite d'un jugement où il y a SDC, l'équipe É-O oriente le cas vers l'équipe de prise en charge.

Afin d'illustrer la grande prudence qui s'impose dans l'interprétation des statistiques actuelles sur la prévalence, nous avons évalué les statistiques disponibles des rapports annuels des DPJ, de 1990 à 1993. Le nombre d'évaluations de cas d'abus sexuels menées à terme dans l'ensemble du Québec sur trois ans était, pour chacune des années, 2974 (1990-1991), 3023 (1991-1992) et 3687 (1992-1993). On pourrait conclure qu'il y a eu une augmentation significative du nombre d'abus sexuels en considérant le nombre des évaluations effectuées; toutefois, ce chiffre ne révèle pas quel pourcentage de cas était jugé fondé. Au cours de ces trois mêmes années, le nombre de cas où des mesures ont été appliquées a baissé : 1449, 1421 et 1227 . Bref, selon les indications, le nombre d'évaluations complétées suggère une augmentation; cependant, le nombre de situations orientées vers I'application des mesures suggère une baisse de la prévalence de l'abus sexuel.

Nous préconisons une grande prudence dans I'interprétation des diverses statistiques disponibles aussi longtemps qu'il n'y aura pas d'uniformité entre les différentes phases décisionnelles ni de recherches scientifiques à une plus grande échelle. De multiples facteurs pourraient 
avoir une influence d'une façon simultanée sur les statistiques de l'incidence de l'abus sexuel, tels que: une augmentation réelle du nombre de personnes abuseures, une fluctuation dans les capacités parentales liée à la crise économique ou à l'augmentation du taux des familles recomposées, une plus grande vigilance du public ayant pour effet d'augmenter le pourcentage des allégations non fondées, une tendance lors de I'É-O à être moins préoccupé par l'abus commis par des tiers, une surcharge des équipes à l'application des mesures, une réussite - au moins partielle - des programmes de prévention, etc.

\section{LE PROFIL PSYCHOSOCIAL DES DIFFÉRENTS ACTEURS DANS L'ABUS SEXUEL}

Les recherches étiopathogéniques recensées se déploient selon trois axes distincts qui traitent respectivement (1) de la nature, de l'ampleur, de l'intensité et de la chronicité des séquelles psychosociales observées chez les victimes d'abus sexuel intrafamilial, (2) de la diversité des caractéristiques sociodémographiques et des troubles psychosociaux notés chez les parents abuseurs et (3) de la multiplicité des structures et des modes d'organisation des relations au sein des familles des victimes d'abus sexuel. Jusqu'à présent, la majorité des études se distinguent par leur caractère athéorique (Brière et Elliott, 1993 ; KendallTackett et al., 1993; Nash et al., 1993). Une analyse des principales contributions met toutefois en évidence la présence de quelques modèles théoriques qui visent principalement à expliquer les séquelles observées chez les victimes. Ainsi la théorie des symptômes distinctifs stipule que les manifestations centrales de l'abus sexuel sont le trouble posttraumatique et la présence de comportements sexualisés (voir Corwin, 1989 ; Jampole et Weber, 1987), alors que les modèles à facettes multiples décrivent l'évolution des conséquences du processus d'abus. Il en est ainsi de celui de Finkelhor et Browne (1985; sexualisation traumatique, trahison, stigmatisation et impuissance) et de celui de Brière (1992; auto-évaluation négative, perception chronique de danger ou d'injustice, impuissance et préoccupation vis-à-vis du contrôle, autoréférence déficiente et réduction des états internes douloureux). Certaines théories, dont celle de Clausen et Crittenden (1991), se centrent davantage sur la présence de dysfonctions familiales conduisant à l'abus. Chacun de ces modèles ne jouit cependant que d'appuis empiriques mitigés et la réflexion théorique devra se poursuivre. 


\section{Les séquelles psychosociales observées chez les victimes d'abus sexuel}

II n'existe pas dans les écrits de consensus sur le type, la sévérité ou la fréquence des séquelles de l'abus, ni sur les facteurs de risque. Sans doute, le faible nombre d'études valides sur le plan scientifique augmente la variation des conclusions sur ces questions. Dans la meilleure recension des écrits publiés jusqu'à maintenant, Kendall-Tackett et ses collègues (1993) révèlent que 45 études scientifiques respectent des critères méthodologiques minimaux (victimes de moins de 18 ans, comparaison d'un groupe d'enfants victimes d'abus sexuel à un groupe d'enfants non victimes d'abus sexuel ou à des normes et quantification des résultats). En ce qui a trait à la nature des symptômes observés chez des victimes d'abus sexuel, ces auteurs établissent deux types de comparaisons : (1) entre des enfants victimes d'abus sexuel et des enfants non victimes d'abus sexuel et (2) entre des enfants victimes d'abus sexuel et des enfants non victimes d'abus sexuel, mais en traitement pour d'autres types de difficultés.

Dans le premier groupe d'études, les résultats font état de différences significatives stables pour la configuration de séquelles suivantes: peur, cauchemar, stress post-traumatique généralisé, comportement de retrait, névrose, cruauté, délinquance, comportement sexuel inapproprié, comportement régressif (énurésie, encoprésie, crises de colère ou pleurnichage), fugue, trouble du comportement et comportement autodestructeur. Mis à part les tentatives suicidaires, la faible estime de soi constitue le symptôme qui différencie le moins bien les victimes d'abus sexuel des enfants non victimes d'abus; la différence entre les deux groupes de sujets n'est significative que dans $50 \%$ des cas.

Dans le second groupe de recherches, le portrait se révèle différent. Ainsi, sauf pour les comportements sexuels inappropriés et le trouble post-traumatique, les enfants victimes $d^{\prime}$ abus sexuel affichent moins de difficultés psychosociales que les enfants en traitement pour $d^{\prime}$ autres types de problèmes. Cette conclusion appelle toutefois deux nuances importantes. Tout d'abord, rien n'assure que les clientèles cliniques dites non abusées ne contiennent pas des enfants victimes d'un abus sexuel qui n'a pas été détecté. De plus, les enfants du second groupe ont été envoyés en clinique parce qu'ils présentaient des comportements symptomatiques particuliers. Il est possible que ces études $\mathrm{n}^{\prime}$ incluent pas plusieurs sous-groupes de victimes: a) des victimes montrant des séquelles seulement à long terme; b) des victimes ne vivant pas des séquelles importantes; et c) des victimes subjuguées par des abuseurs qui les empêchent de les dénoncer. 
Bien que la plupart des études n'aient pas contenu suffisamment de détails pour qu'ils procèdent à des méta-analyses, Kendall-Tackett et ses collègues (1993) présentent des statistiques détaillées sur le pourcentage de victimes d'abus sexuel souffrant de symptômes spécifiques selon la catégorie d'âge à laquelle elles appartiennent. Quelques observations générales ressortent de ces analyses. Il apparaît d'abord que le pourcentage de victimes affichant un symptôme particulier dépasse les $40 \%$ uniquement dans le cas du trouble post-traumatique généralisé, où le pourcentage se situe à $53 \%$. Autrement, le pourcentage de victimes souffrant d'un symptôme varie de $20 \%$ à $30 \%$. Lorsque les données sont catégorisées en fonction de trois catégories générales d'âge (âge préscolaire : 0 à 6 ans; âge scolaire: 7 à 12 ans; adolescence: 13 à 18 ans), les résultats montrent certaines trajectoires développementales spécifiques dans les séquelles de l'abus sexuel. Par exemple, chez les enfants d'âge préscolaire, l'anxiété, les cauchemars, le trouble post-traumatique, l'internalisation, l'externalisation et les comportements sexuels inappropriés constituent les symptômes les plus fréquents. En ce qui a trait aux enfants d'âge scolaire, les symptômes les plus communs sont la peur, la névrose, l'agressivité, les cauchemars, les troubles scolaires, l'hyperactivité et les comportements régressifs. Enfin, chez les adolescents, ce sont la dépression, le retrait, les idées suicidaires, les comportements autodestructeurs, les troubles somatiques, les actes délictueux, la fugue et la toxicomanie. Certains symptômes paraissent relativement robustes peu importe la catégorie d'âge. Ainsi, la dépression et les difficultés d'apprentissage semblent présentes chez les trois groupes de victimes. Par ailleurs, d'autres symptômes se transforment selon une séquence développementale prévisible. Les comportements antisociaux de l'âge préscolaire et scolaire deviennent les comportements délictueux d'adolescence, tandis que les comportements sexuels inappropriés semblent disparaître à la période de latence pour réapparaître à l'adolescence sous la forme de promiscuité, de prostitution ou d'agression sexuelle.

Une excellente étude récente, menée sur 63 enfants victimes d'abus sexuel suivis au CPEJ de Lanaudière et sur des enfants du système scolaire normal (Tourigny, Péladeau et Doyon, sous la direction de C. Bouchard, 1993), a révélé des résultats intéressants : avant l'intervention, "...les enfants abusés sexuellement présentent [...] une santé psychologique légèrement plus détériorée: ils sont plus soumis et moins affirmatifs, leur lieu de contrôle est plus externe, ils sont plus dépressifs et se perçoivent comme moins compétents que le groupe d'enfants de la région de Lanaudière. À l'exception de la soumission et de l'agression, ces différences sont toutefois légères, $c^{\prime}$ est-à-dire qu'elles représentent un maximum de dix percentiles. » (Tourigny et al., 1993: vii). 
L'analyse de Kendall-Tackett et de ses collègues (1993) fait aussi ressortir la présence d'un pourcentage élevé d'enfants asymptomatiques. Ce pourcentage varie de $20 \%$ à $60 \%$ selon les études et il peut s'expliquer de trois façons: I'absence de sensibilité des instruments de mesure utilisés par les chercheurs, I'hypothèse d'un délai dans l'apparition des séquelles psychosociales et la possibilité que ces enfants soient réellement moins troublés que les autres par l'abus sexuel. En fait, ces trois sources d'explication sont possiblement complémentaires. Jusqu'à présent, les chercheurs n'ont pas accordé suffisamment d'intérêt à l'étude de ce phénomène et de ses corrélats (Van Gijseghem et Gauthier, 1992).

Un débat est en cours relativement à l'impact du lien entre l'abuseur et la victime et aux séquelles de l'abus. Il existe très peu d'études qui traitent strictement des séquelles psychosociales observées chez les victimes d'abus sexuel intrafamilial. Kendall-Tackett et ses collègues (1993) révèlent que, parmi les 45 études, dix seulement établissent des distinctions entre les situations d'abus sexuel intrafamilial et extrafamilial. L'analyse comparative de ces résultats démontre que dans sept des dix cas les répercussions de l'abus sexuel sont plus sévères chez des victimes d'abus sexuel intrafamilial que chez des victimes d'abus sexuel extrafamilial. Ces débats portent sur la nature, I'ampleur, I'intensité et la chronicité des séquelles psychosociales observées chez les victimes d'abus sexuel intrafamilial. Le bilan présenté par Kendall-Tackett et ses collègues peut cependant servir de toile de fond à une discussion préliminaire de ces questions. Il faut toutefois garder à l'esprit qu'à cause des différences notées entre les séquelles de l'abus sexuel intra et extrafamilial, il est possible que cette extrapolation conduise à une sousestimation des séquelles de l'abus sexuel intrafamilial.

La nature, I'ampleur, l'intensité et la chronicité des séquelles de l'abus sexuel sont probablement liées au jeu complexe de divers facteurs, tels l'âge de l'enfant au moment de l'évaluation et au moment de l'événement traumatique, le sexe de l'enfant, la sévérité de l'abus, la fréquence de l'abus, la durée de l'abus, I'utilisation de la force, le lien entre la victime et l'abuseur, le soutien du ou des parents non abuseurs, le laps de temps écoulé depuis le dernier incident et les mécanismes d'adaptation de l'enfant.

Cette courte présentation des séquelles psychosociales associées à l'abus sexuel doit être interprétée à la lumière de plusieurs faiblesses méthodologiques des études colligées (Sabourin, Wright, Lussier et Bélanger, 1993). Ainsi, les faiblesses suivantes ressortent: le caractère athéorique des recherches, la petite taille des échantillons, le recours à des instruments de mesure non standardisés et peu sensibles, I'utilisation de données ne provenant que d'une seule des sources d'évaluation possibles (I'enfant, les parents ou les cliniciens), la différenciation des 
résultats selon l'âge et le sexe des victimes, l'ignorance et l'absence de contrôle de facteurs intermédiaires cruciaux et le recours à des stratégies d'analyse statistique simplistes ou erronées.

\section{Les caractéristiques du parent abuseur}

Dans la majorité des cas d'abus sexuel intrafamiliaux (plus de $90 \%$; Tourigny, 1991), le père se révèle être le parent abuseur. La recension des écrits traite donc presque uniquement des pères abuseurs. Un examen attentif de la trentaine d'études portant sur ce thème fait ressortir les conclusions qui suivent (Abel et Rouleau, 1990; Meyer Williams et Finkelhor, 1990). Tout comme dans le cas des séquelles observées chez les victimes d'abus, il n'existe pas une configuration spécifique de caractéristiques sociodémographiques, psychosociales ou psychophysiologiques communes à l'ensemble des pères abuseurs. Par contre, lorsque le profil de fonctionnement de ces pères est comparé à celui $d^{\prime}$ 'autres groupes d'hommes, les chercheurs rapportent que (1) près de la moitié des pères abuseurs ont subi diverses formes de mauvais traitements durant leur enfance et près de $20 \%$ ont fait l'objet d'abus sexuel avant 18 ans; ils rapportent fréquemment s'être sentis rejetés par leur père; (2) les pères abuseurs identifiés présentent aussi des traits paranoïdes et ils souffrent $d^{\prime}$ isolement social; certains chercheurs parlent $d^{\prime}$ 'une structure de personnalité psychopathique; (3) les pères abuseurs recrutés sont généralement passifs et ils éprouvent des troubles d'identité personnelle et sexuelle; ils s'identifient peu aux aspects traditionnels du rôle masculin; (4) plusieurs de ces pères abuseurs rapportent un degré élevé de détresse conjugale; ils sont peu stimulés sexuellement, voire dégoûtés, par un partenaire sexuel adulte et ils éprouvent dans une proportion de $20 \%$ à $35 \%$ une attirance sexuelle déviante à l'endroit des enfants ; (5) les pères abuseurs identifiés montrent une faible capacité d'empathie, surtout à l'égard des enfants, et ils affirment ne pas s'être impliqués activement sur le plan des soins à prodiguer à leurs enfants en bas âge ; leur capacité d'attachement semble donc déficitaire et une dynamique de désinvestissement du père semble à l'œuvre et enfin ; (6) près de la moitié des pères qui sont impliqués dans des situations d'abus sexuel intrafamilial sont aussi impliqués dans des situations d'abus sexuel extrafamilial.

En résumé, les études empiriques les plus récentes montrent la présence de facteurs étiologiques multiples liés à l'adoption de conduites abusives. La diversité marquée des causes potentielles énumérées plus haut, juxtaposée à l'existence d'un grand nombre de pères abuseurs 
qui ne participent pas aux recherches, laisse présager la nécessité de circonscrire plusieurs types de parents abuseurs.

La validité et la pertinence clinique de ces conceptions multidimensionnelles devront cependant être examinées sur le terrain. De plus, ces typologies devront être étudiées à la lumière de la convergence entre les profils des parents abuseurs et les caractéristiques du fonctionnement psychologique et social des enfants victimes d'abus; certains enfants constituent en effet des sujets à risque élevé à cause, par exemple, de leur âge ou de leur isolement social. L'établissement de configurations multidimensionnelles qui respecteront la complexité de la réalité clinique nécessitera le recours à des évaluations multimodales tels l'entrevue structurée, les questionnaires standardisés et les techniques psychophysiologiques usuelles. Enfin, les chercheurs et les intervenants doivent déterminer dans quelle mesure ces portraits ne représentent que le reflet des conséquences de la divulgation de l'abus plutôt que le reflet des causes à l'origine des comportements sexuels abusifs. Ces différences potentielles pourront toutefois être contrôlées en obtenant de la mère et de la personne victime $d^{\prime}$ 'abus une description du père abuseur et en comparant les profils observés en fonction de la source des données.

Notre présentation des résultats de recherche spécifiant les caractéristiques biopsychosociales des pères qui abusent de leurs enfants doit, encore une fois, être interprétée à la lumière de plusieurs faiblesses méthodologiques des études colligées. Aux limites déjà présentées dans d'autres sections, il faut ajouter la propension des pères abuseurs à rapidement rapporter qu'ils sont «guéris ». Peu de chercheurs ont tenté d'évaluer l'effet de ce biais, de le minimiser ou de le contourner.

\section{Les caractéristiques des mères}

La lecture de la documentation clinique produite au cours des trente dernières années sur les caractéristiques des mères des victimes d'abus sexuel intrafamilial fait ressortir les observations suivantes: (1) pour certains, la mère pousserait consciemment ou non sa fille à se laisser victimiser, (2) la mère ne serait pas capable de s'impliquer vis-à-vis de son conjoint sur le plan affectif et sexuel, ce qui entraînerait ce dernier à satisfaire ses besoins autrement (Elbow et Magfield, 1991), (3) la mère serait, autant que l'enfant, une victime passive du pouvoir dictatorial de son conjoint (Herman, 1988) et (4) la mère se révèle incapable de protéger sa fille, parce qu'elle craint les représailles physiques, psychologiques ou économiques de son conjoint (Dietz et Craft, 1980). 
En dépit de ce portrait, les résultats des quelques recherches descriptives sur le sujet sont inconsistants. Ainsi, Groff (1987) prétend noter des élévations significatives sur les échelles de sociopathie et de névrose du MMPI, tandis que Wagner (1991) n'a pas relevé de différences entre les mères des victimes et des mères qui consultent pour d'autres motifs. Enfin, les travaux de Hubbard (1989) indiquent la présence, chez les mères des victimes d'abus sexuel intrafamilial, d'un degré élevé de détresse psychologique et de confusion; il a aussi observé des symptômes qui se rapprochent de ceux qui découlent du trouble post-traumatique. Ces études n'échappent cependant pas aux nombreuses critiques méthodologiques énumérées précédemment. En conclusion, il apparaît donc que les nombreuses hypothèses sur les caractéristiques des mères des victimes d'abus sexuel intrafamilial n'ont pas été évaluées de façon scientifique.

\section{STRUCTURES ET MODES D'ORGANISATION DES RELATIONS FAMILIALES}

Depuis plus d'une décennie, des intervenants et des chercheurs de plusieurs disciplines soulignent la multiplicité des structures et des modes d'organisation des relations au sein des familles où se produit l'abus sexuel. Ainsi note-t-on la présence de structures familiales chaotiques ou à l'inverse hyper-rigides, la faible différenciation des structures identitaires des individus, l'inversion des rôles mère-fille et la répartition inégale du pouvoir au sein de la famille: dominance matriarcale ou patriarcale (Giarretto, 1982 ; Levitt, Owen et Truchess, 1991 ; Paradis, Perron et Dubé, 1992). En outre, la divulgation de l'abus sexuel peut provoquer de profonds bouleversements familiaux, dont l'éclatement du couple (Burgess, Holmstrom et McCausland, 1977 ; Sirles et Lofberg, 1990). Malheureusement, la richesse de ces observations ne s'appuie pas sur un grand nombre d'études empiriques rigoureuses. De plus, encore ici, il apparaît difficile de départager les propriétés systémiques attribuables au dévoilement de l'abus sexuel intrafamilial de celles associées à l'éclosion et au maintien des situations d'abus.

L'analyse des facteurs de vulnérabilité familiaux potentiellement associés au développement de l'abus sexuel s'appuiera ici sur une classification en quatre catégories: la transmission intergénérationnelle de l'abus intrafamilial, les caractéristiques structurales des systèmes familiaux où le père est abuseur, les processus communicationnels à I'œuvre au sein de ces familles et l'environnement social propice au développement des situations d'abus sexuel. 
La transmission intergénérationnelle de l'abus sexuel intrafamilial est un thème fréquemment abordé dans les discussions cliniques. En fait, la présence d'abus sexuels au sein de la famille d'origine est souvent considérée comme un facteur de risque important (Barrett, Sykes et Byrnes, 1986 ; Carson et al., 1991). Toutefois, il n'existe pas, à notre connaissance, de recherches empiriques sur la prévalence de ce phénomène.

L'étude approfondie des propriétés structurales des familles où des situations d'abus sexuel prévalent fait ressortir les observations suivantes. En premier lieu, plusieurs chercheurs constatent la présence de frontières intergénérationnelles floues entre les systèmes familiaux d'origine des parents et le système familial des individus où l'abus sexuel se déroule (Carson et al., 1991). Cette caractéristique constitue d'ailleurs une condition probablement préalable, voire essentielle, à la transmission intergénérationnelle de l'abus sexuel. Dans cette même veine, Alexander (1985) et Ribordy (1989) ont constaté l'absence de frontières interpersonnelles étanches entre les membres d'une famille où se produit I'abus sexuel. Ces frontières floues engendrent des états confusionnels où les parents se conduisent comme des pairs pour les enfants et non comme des figures d'autorité soutenantes (Barrett, Sykes et Byrnes, 1986). Au surplus, cette confusion se répercute sur la faible différenciation des structures identitaires des membres de la famille, sur le peu d'individuation entre les personnes et sur le soutien accordé à l'autonomie et à la vie privée des enfants par les parents (Trepper et Sprenkle, 1988; Woodworth, 1991). Cependant, les relations entretenues avec les autres familles sont marquées par la présence de frontières nettes et bien délimitées (Alexander, 1985 ; Herman, 1988). L'inversion des rôles mèrefille dans diverses sphères, telles les tâches ménagères, l'éducation des enfants et éventuellement la sexualité, représente aussi un facteur commun aux familles où se développe I'abus sexuel.

Enfin, en ce qui concerne la structure des relations de pouvoir entre les parents, les chercheurs et les cliniciens rapportent la présence de deux modes d'organisation hiérarchiques opposés: la structure patriarcale et la structure matriarcale. Dans le premier cas, le père adopte des attitudes et des comportements contrôlants à l'égard de sa femme et de ses enfants; la mère se révèle incapable $d$ 'assurer la protection des enfants (Barrett, Trepper et Fish, 1990). Dans le second cas, la mère adopte des conduites dominantes empreintes de froideur et de rejet; le répertoire comportemental du père est alors marqué par la dépendance, I'infantilisation et le recours à des substances qui augmentent faussement son sentiment de contrôle (Ribordy, 1989 ; Trepper et Sprenkle, 1988). Les structures familiales hyper-rigides semblent les plus fréquentes. Les 
membres de ces systèmes adhèrent à un ensemble de règles strictes même si celles-ci sont irrationnelles et destructrices; les stéréotypes sexuels y sont très accentués. Les structures familiales chaotiques se caractérisent, à l'inverse, par l'absence de règles stables clairement définies; les parents ne possèdent pas les habiletés parentales nécessaires pour implanter et gérer des normes de fonctionnement harmonieux.

L'analyse des processus communicationnels différenciant les familles où se produit l'abus sexuel des familles «normales» fait ressortir (1) le caractère indirect des échanges, (2) la présence de méfiance, d'hostilité contenue, de secrets et de doubles contraintes, (3) l'évitement systématique des conflits de nature affective et l'absence d'intimité, (4) I'absence d'habiletés de résolution de problèmes efficaces, (5) le développement de difficultés générales d'ordre sexuel et (6) la résistance systématique au changement (Alexander, 1985 ; Carson et al., 1991 ; Lang et al., 1990). Larson et Maddock (1986) ont poussé cette étude des compétences communicationnelles un peu plus loin en montrant que les échanges fluctuent selon la gravité de l'abus. Enfin, Gupta et Cox (1988) ont élaboré une typologie des transactions communicationnelles s'appuyant sur une conception psychodynamique du fonctionnement du père abuseur.

L'environnement social propice au développement des situations $d^{\prime}$ 'abus sexuel n'a certes pas fait l'objet de multiples recherches. Toutefois, il semble que le degré de tolérance à l'abus sexuel dans l'entourage immédiat, l'acceptation généralisée de la suprématie masculine traditionnelle, la présence de stress sociaux perturbateurs contribuent à l'éclosion de situations d'abus sexuel intrafamilial (Barrett, Trepper et Fish, 1990; Herman, 1988 ; Trepper et Sprenkel, 1988).

Il faut aussi mentionner que la divulgation de l'abus sexuel entraîne parfois l'éclatement de la famille. Ainsi, les résultats des recherches menées par Burgess et ses collègues (1977), de même que par Sirles et Lofberg (1990), révèlent que près de $50 \%$ des situations d'abus résultent en une séparation ou un divorce. L'étude de Sirles et Lofberg (1990) constitue sans contredit la meilleure étude sur le sujet. Ces derniers ont recruté 128 familles au sein desquelles l'abuseur est le père biologique $(n=58)$ ou le conjoint de la mère (beau-père ou concubin; $n=70$ ). Les couples qui se séparent ou qui divorcent à la suite de la divulgation de l'inceste se distingueraient des couples qui poursuivent leur relation sur les dimensions suivantes: la victime est plus jeune, elle dévoile l'abus à sa mère plutôt qu'à une amie ou à un professionnel et la mère la croit enfin, la famille est aux prises avec d'autres types 
de violence et le laps de temps écoulé depuis le début de l'abus s'avère plus court. L'éclatement du couple n'est pas lié au statut marital du père (père biologique ou concubin), au statut d'emploi de la mère, à la violence physique subie par l'enfant, au déni de l'abus par le père, à la nature des actes sexuels commis et à la consommation de substances psychotropes par le père.

Sur le plan méthodologique, l'ensemble des recherches évaluatives comporte les mêmes lacunes que celles observées dans les recherches citées précédemment sur les séquelles psychosociales observées chez les victimes.

\section{DÉFIS DE L'ÉVALUATION DE L'EFFICACITÉ DE LA RÉPONSE INSTITUTION NELLE}

Il est plus exigeant de mener des recherches sur l'efficacité de la réponse institutionnelle que de mener des recherches descriptives. II ne faut pas seulement établir une évaluation compréhensive de chaque membre de la famille visée par l'intervention, encore faut-il aussi : (1) mesurer les différents paramètres de l'intervention et composer avec la réalité selon laquelle la majorité des clients des Centres jeunesse reçoivent plus d'une intervention (Tourigny et al., 1993; Wright, Paradis, Pinard, Perron et Gagnon, 1995); (2) évaluer le client après l'intervention ; (3) déterminer si le client a effectivement terminé le traitement et si les caractéristiques des clients qui terminent le traitement sont différentes de celles des clients qui abandonnent prématurément; (4) évaluer le changement à court et à long terme; et (5) estimer le degré de changement attribuable au traitement (en respectant les codes déontologiques), soit par un groupe de sujets-cohorte ou par la comparaison entre deux formes d'intervention. Ainsi qu'il a été mentionné dans d'autres recensements (Becker, 1994 ; Beutler et al., 1994; Wright, Sabourin, Lussier et Bélanger, 1995), les recherches évaluatives qui respectent des critères scientifiques sont plus rares que les études descriptives recensées précédemment. À notre avis, l'étude pionnière de Tourigny, Péladeau et Doyon (1993) met en évidence le risque que les intervenants tiennent pour acquis que tous les clients répondent positivement aux services offerts au Centre jeunesse. Elle souligne également le danger que les chercheurs concluent trop rapidement sur l'efficacité générale des interventions sans tenir compte des problèmes d'attrition sélective des clients (les clients qui vont bien peuvent terminer plus tôt) et des échantillons trop restreints. 


\section{DÉFIS DE LA RECHERCHE DESCRIPTIVE EN MATIĖRE D'ABUS SEXUEL EN VERS LES ENFANTS}

Le précédent recensement des écrits et une première analyse des priorités des intervenants de l'actuel partenariat (Wright et al., 1994) soulignent la pertinence de mener des études descriptives sur les multiples acteurs associés à l'abus. De solides données dans ce secteur pourraient nourrir: (1) le processus d'évaluation du signalement; (2) les décisions sur la nécessité de passer à la phase traitement; (3) le choix des mesures et la nécessité d'éloigner la victime de l'abuseur; (4) le choix des cibles de l'intervention psychosociale; (5) l'établissement des facteurs de risque et de protection; et 6) les outils pour évaluer le statut du client avant et après les interventions, afin d'établir l'efficacité de celles-ci.

Plusieurs décisions stratégiques s'imposent au moment de la planification des recherches descriptives dans ce secteur. Nous en aborderons quatre ici. Une première préoccupation consiste à déterminer le point d'entrée optimal d'administration d'une batterie d'évaluation du fonctionnement psychosocial des différents acteurs engagés dans cette chaîne complexe d'événements. D'un strict point de vue scientifique, nous avons déjà précisé que la représentativité de l'échantillon augmente non seulement en fonction de la taille de l'échantillon, mais surtout en fonction du moment où les instruments de mesure sont administrés: plus la recherche intervient tardivement et plus l'échantillon est biaisé. De ce point de vue, le meilleur point d'entrée se situera donc au niveau soit du RTS, soit de l'É-O. Cependant, pour des raisons éthiques, l'ensemble des données sur les cas de faits non fondés doit être détruit dès que la décision est adoptée. Le scénario possible consiste en une collecte des données descriptives auprès de chaque personne associée à la phase "Évaluation»; seulement les données sur les cas de FF seront retenues. Cependant, toute étude tentant d'éclairer la façon dont les intervenants arrivent à la décision sur la présence d'abus et une estimation des taux d'erreurs ( «ausse négative») deviennent plus difficiles. Sur le plan de l'éthique et des coûts des ressources humaines, c'est à l'étape «Orientation», une fois que l'abus a été confirmé, qu'il sera le plus facile d'introduire les mesures. Ce moment permettra la comparaison de plusieurs groupes selon les décisions prises par les évaluateurs, telles: sécurité compromise comparativement à non compromise, mesures volontaires versus judiciarisation. Un suivi des taux de récidive de ces groupes favorisera la pertinence des données descriptives, afin de faire avancer les recherches sur les processus décisionnels des évaluateurs et sur les facteurs de risque et de protection. 
Une deuxième série de préoccupations consiste à déterminer qui on doit évaluer et avec quel instrument. À la première question, il y a de multiples avantages à évaluer non seulement la victime, mais également l'abuseur (indépendamment du lien avec la victime), le ou les parents non abuseurs, les modes d'organisation des relations familiales et l'environnement sociofamilial. La batterie d'instruments nécessaires devra respecter les critères les plus exigeants de la tradition scientifique en psychologie clinique, en travail social et en évaluation de programmes. À ce stade-ci, la pertinence clinique des instruments constitue le critère le plus important. En effet, l'adoption d'un tel critère optimisera les possibilités de collaboration entre les intervenants et les chercheurs. Les propriétés psychométriques des instruments de mesure représentent un deuxième critère de sélection important. En effet, outre la fidélité des scores, il importe de choisir des instruments de mesure qui (1) discriminent bien les cas d'abus sexuel des autres cas d'abus, (2) comportent des dimensions d'évaluation propres à l'abus sexuel, (3) couvrent d'autres secteurs jugés pertinents mais non directement reliés à l'abus sexuel, (4) soient disponibles en langue française ou qui pourraient être traduits par les membres de notre équipe. Enfin, (5) le choix des instruments de mesure sera fonction de leurs coûts d'administration et de correction. Ce dernier facteur dépend directement de la qualité du financement disponible pour mener à terme ces projets de recherche.

L'évaluation des caractéristiques de la personne abuseure nécessitera aussi I'analyse approfondie des instruments qui permettent de quantifier des variables telles que l'histoire personnelle et sexuelle (Nichols et Molinder, 1984), les fantasmes et les préférences sexuelles (Abel et Rouleau, 1990), les compétences sociales (Whitman et Quinsey, 1981), les distorsions cognitives (Abel et Rouleau, 1990), la minimisation et le déni (Barbaree, 1991) et la capacité d'empathie (Davies, 1980). Certains instruments pourront être remplis par la personne abuseure et d'autres, telle l'échelle d'évaluation du risque de récidive (McGovern et Peters, 1988), seront remplis par l'intervenant.

L'évaluation des caractéristiques du père et de la mère devra s'appuyer aussi sur des dimensions fréquemment mentionnées dans la documentation: I'estime de soi (Vallerand et Vallières, 1990), la détresse psychologique (Tousignant et Kovess, 1985), la satisfaction conjugale (Baillargeon et al., 1986) et la satisfaction sexuelle (Hudson, 1982 ; Wright, Sabourin, Turcotte, McDuff et Fichten, 1993). Quant au profil de la mère, certaines hypothèses avancées dans les écrits pourront être examinées à l'aide d'instruments de mesure de l'affirmation de soi, de l'histoire familiale, du soutien à l'enfant victime d'abus, 
du style d'attachement au conjoint et à l'enfant abusé (Hazan et Shaver, 1987 ; Lussier, 1991a), des mécanismes d'adaptation (Endler et Parker, 1990 ; Lussier, 1991b) et du déni (Paulhus, 1984; Sabourin et al., 1989).

Du côté de la famille, le Family Functioning Scale (Bloom et Lipetz, 1987) mesure 15 dimensions, dont 9 se sont avérées pertinentes pour distinguer les victimes d'abus (Nash et al., 1993). Le Family Environment Scale (Moos et Moos, 1986) est aussi très fréquemment utilisé. L'observation directe du style d'interactions familiales, telles la dominance, la communication, l'expression de l'affection, la compétence parentale et la résolution de problèmes, quoique très coûteuse en ressources humaines et financières, sera nécessaire afin d'évaluer adéquatement plusieurs hypothèses citées précédemment sur les différences entre les familles des enfants victimes d'abus sexuel intra versus extrafamilial et les familles des enfants non victimes d'abus.

Une troisième préoccupation découle de la masse de renseignements qui devra être colligée et entreposée de façon uniforme entre les différents sites qui collaborent à la recherche. Ce défi peut être relevé par des systèmes de tenue de dossiers de plus en plus conviviaux (user friendly) et de moins en moins coûteux. La création d'une banque de données scientifiques comprenant des renseignements sociodémographiques et psychosociaux sur les situations d'abus sexuel pour chacun des territoires couverts par la recherche aura des retombées administratives favorables. De plus, I'informatisation des données de recherche vise à accélérer la communication entre les chercheurs et les intervenants. Toutefois, l'examen des avantages et des inconvénients à communiquer directement les résultats obtenus par leurs clients aux intervenants responsables du dossier constitue le quatrième thème de discussion important. En effet, idéalement, sur le plan clinique, l'intervenant devrait avoir accès à un résumé intelligible des conclusions qui ressortent des instruments de mesure à l'intérieur d'une semaine, avec beaucoup de succès. La transmission rapide des résultats augmente la collaboration des intervenants et peut améliorer la qualité de l'application des mesures. Toutefois, sur le plan scientifique, certains auteurs prétendent que l'adoption de telles pratiques risque d'entacher la validité interne des protocoles de recherche. Ces objections ont cependant moins de valeur si la démarche de communication des résultats aux intervenants est maintenue même lorsque les recherches évaluatives cessent. 


\section{CONCLUSION}

Une évaluation initiale des priorités des intervenants des cinq Centres jeunesse membres du PRIMASE révèle une grande ouverture aux recherches descriptives et évaluatives en matière d'abus sexuel. On constate également, à l'heure actuelle, une grande volonté de l'ensemble des établissements Centres jeunesse de tenter de définir les pratiques les plus efficaces. À titre d'exemple, en novembre et décembre 1994, un bilan des pratiques a été dressé dans tous les Centres de protection de l'enfance et de la jeunesse du Québec, à partir de quatre instruments. Ce bilan, dont l'initiative revient à l'Association des centres jeunesse du Québec, examine plusieurs dimensions des pratiques tels les critères de rétention des signalements, la classification des cas d'abus, les modes de pratique à l'Évaluation et l'Orientation, les modes de pratique concernant les plaintes policières et une foule de renseignements en ce qui a trait aux réponses institutionnelles en matière d'abus. Les résultats, qui devraient être disponibles en septembre 1995, nourriront des réflexions sur les modifications à apporter dans ce secteur de la protection de la jeunesse et faciliteront l'établissement des priorités de recherche.

Nous pouvons souhaiter qu'au Québec, en dépit de la conjoncture économique, divers groupes d'intervenants, plusieurs équipes de recherche et les décideurs se concerteront afin d'offrir la meilleure réponse possible aux victimes et à toute autre personne affectée par l'abus sexuel auprès des enfants. Dans une récente prise de position très pertinente (Sexual Abuse of Chidren, 1994 - voir Beutler et al., 1994), aux États-Unis, plusieurs des chefs de file dans ce secteur ont souligné que ce type de concertation qui traverse plusieurs frontières professionnelles et institutionnelles est essentiel si nous voulons être en mesure de combler des manques flagrants dans les pratiques et les recherches existantes.

\section{Références bibliographiques}

ABEL, G.G. et J. ROULEAU (1990). "The nature and extent of sexual assault», dans W.L. Marshall, D.R. Laws et H.E. Barbaree (dir.), Handbook of Sexual Assault. New York: Plenum.

AlEXANDER, P.C. (1985). "A systems theory conceptualization of incest », Family Process, $24: 79-88$. 
Badgley, R.F., H. Allard, N. McCormick, P. Proodfoot, D. Fortin, D. OGilvie, Q. RaeGrant, P. GÉlinas, L. PÉPIN et S. Sutherland (1984). Infractions sexuelles à l'égard des enfants. Ottawa: Approvisionnements et Services Canada.

BAGLEY, C. et R. RAMSEY (1986). "Sexual abuse in childhood: Psychosocial outcomes and implications for social work practice», Journal of Social Work and Human Sexuality, vol. 4 : 33-47.

BAILLARGEON, J., G. DubOIS et R. MARINEAU (1986). Traduction française de l'échelle d'ajustement dyadique, Revue canadienne des sciences du comportement, vol. 18:25-34.

Barbaree, H.E. (1991). The Denial and Minimization Checklist. Kingston, Ontario: The Wackworth Sexual Behavior Clinic.

BARRETT, M.J., C. SYKES et W. BYRNES (1986). «A systemic model for the treatment of intrafamily child sexual abuse », Journal of Psychotherapy and the Family, vol. 2, no $2: 67-82$.

BARRETT, M.J., T.S. TREPPER et L.S. FISH (1990). «Feminist-informed family therapy for the treatment of intrafamily child sexual abuse », Journal of Family Psychology, vol. 4, $\mathrm{n}^{\circ}$ 2:151-166.

BECKER, J.V. (1994). "Offenders: Characteristics and Treatment», Sexual Abuse of Chidren, The Future of Children, vol. 4, $\mathrm{n}^{\circ} 2: 176-198$.

BEUTLER, L.E., R.A WILLIAM et H.A. ZeTER (1994). "Efficacy of treatment for victims of child sexual abuse ", Sexual Abuse of Children, The Future of Children, vol. 4, $\mathrm{n}^{\circ} 2$ : 156-175.

BLOOM, B. et M. LIPETZ (1987). Revisions on the self-report measure of family functioning (Tech. Rep. no. 2). Boulder: University of Colorado, Center for Family Studies.

BRIÈRE, J. (1992). Child abuse trauma: Theory and treatment of the lasting effects. Newbury Park, CA : Sage.

BRIÈRE, J. et D.M. ElLIOTt (1993). "Sexual abuse, family environment, and psychological symptoms: On the validity of statistical control », Journal of Consulting and Clinical Psychology, vol. 61 : 284-288.

BURGESS, A.W., L.L. HOLMSTROM et M.P. MCCAUSLAND (1977). "Child sexual assault by a family member: Decisions following disclosure », Victimology: An International Journal, vol. 2 : 236-250.

Carson, D.K., L.M. Gertz, M.A. DOnaldSOn et S.A. WONDERLiCH (1991). "Intrafamilial sexual abuse: Family-of-origin and family of procreation characteristics of female adult victims », The Journal of Psychology, vol. $125, \mathrm{n}^{\circ} 5: 125(5), 579-597$.

Clausen, A.H. et P.M. CRittenden (1991). "Physical and psychological maltreatment : relations among types of maltreatment », Child Abuse and Neglect, vol. $15: 5-18$.

Commission de protection des droits de la jeunesse (CPDJ) (1990-1993), Rapport d'activités 1989-1990. Québec: Publications du Québec.

CORWIN, D.L. (1989). "Early diagnosis of child sexual abuse: Diminishing the lasting effects », dans G.E. Wyatt et G.J. Powell (dir.), Lasting effects of child sexual abuse. Newbury Park, CA : Sage, p. 251-270.

DAVIES, M.H. (1980). "A multidimensional approach to individual differences in empathy ", JSAS Catalogue of Selected Documents in Psychology, vol. $10: 85$. 
DieTZ, C. et J. CRAFT (1980). "Family dynamics in incest: A new perspective», Social Casework, 61: 602-609.

ECKenrode, J., J. POWers, J. Doris, J. MunsCH et N. Bolger (1988). "Substantiation of child abuse and neglect reports », Journal of Consulting and Clinical Psychology, 56:9-17.

ELBOW, M. et J. MAYFIELD (1991). "Mothers of incest victims: Villains, victims, or protectors? », Families in Society: The Journal of Contemporary Human Services, 9: 78-86.

ENDLER, N.S. et D.A. PARKer (1990). "Multidimensional assessment of coping: A critical analysis», Journal of Personality and Social Psychology, $58: 844-854$.

FINKELHOR, D. et A. BROWNE (1985). «The Traumatic Impact of Child Sexual Abuse. A Conceptualization», Journal of Orthopsychiatry, vol. 55, $\mathrm{n}^{\circ} 4: 530-541$.

FINKELHOR, D. (1994). "Current information on the scope and nature of child sexual abuse », Sexual Abuse of Children, The Future of Children, vol. 4, $\mathrm{n}^{\circ} 2: 31-53$.

GiarRetTO, H. (1982). "A comprehensive child sexual abuse treatment program», Child Abuse \& Neglect, vol. 6 : 263-278.

GROFF, M.G. (1987). "Characteristics of incest offenders' wives », Journal of Sex Research, 23 : 91-96.

GUPTA, G.R. et S.M. COx (1988). "A typology of incest and possible intervention strategies » Journal of Family Violence, vol. 3, $\mathrm{n}^{\circ} 4: 299-312$.

HAZAN, C. et P. SHAVER (1987). «Romantic love conceptualized as an attachment process », Journal of Personality and Social Psychology, 52 : 511-524.

HERMAN, J.L. (1988). «Father-Daughter incest», dans F.M. Ochenberg (dir.), Post-traumatic therapy and victims of violence. New York: Brunner/Mazel, p. 175-195.

HUBBARD, G.B. (1989). "Mother's perceptions of incest: Sustained disruption and turmoil», Archives of Psychiatric Nursing, vol. iii, $\mathrm{n}^{\circ} 1: 34-40$.

HuDSON, W.W. (1982). The clinical measurement package: A field manual. Homewood, II: Dorsey Press.

JAMPOLE, L. et M.K. WEBER (1987). "An assessment of the behavior of sexually abused and nonsexually abused children with anatomically correct dolls ", Child Abuse \& Neglect, vol. 11: 187-192.

KENDALL-TACKETT, K.A., L.M. WILLIAMS et D. FINKELHOR (1993). "Impact of sexual abuse on children: A review and synthesis of recent empirical studies », Psychological Bulletin, vol. 113, $\mathrm{n}^{\circ} 1:$ 164-180.

LANG, R.A., R. LANGEVIN, V. VAN SANTEN, D. BILlingsLey et P. WRIGHT (1990). "Marital relations in incest offenders ", Journal of Sex and Marital Therapy, vol. 16, no $4: 214-229$.

LAROUCHE, G. (1993). Aux formatrices en intervention auprès des femmes violentées. Montréal : Éditions Wilson et Lafleur.

LARSON, N.R. et J.W. MADDOCK (1986). "Structural and functional variables in incest family systems: Implications for assessment and treatment», Journal of Psychotherapy and the Family, vol. 2, $\mathrm{n}^{\circ} 2: 27-43$. 
LEVITT, C.J., G. OWEN et J. TRUCHESS (1991). «Families after sexual abuse: What helps? What is needed? ", dans M.Q. Patton (dir.), Family sexual abuse: Frontline research and evaluation. Newbury Park, CA : Sage Publications, p. 39-56.

LIPOVSKY, J.A., B.E. SAUNDERS et S.M. MURPHY (1989). "Depression, anxiety, and behavior problems among victims of father-child sexual assault and nonabused siblings ", Journal of Interpersonal Violence, vol. 4, $n^{\circ} 4$ : 452-468.

LUSSIER, Y. (1991a). Traduction française du Adult Attachment Types. Document inédit. Université du Québec à Trois-Rivières.

LUSSIER, Y. (1991b). Traduction française du Multidimensional Coping Inventory. Document inédit. Université du Québec à Trois-Rivières.

MCGOVERN, K. et J. Peters (1988). "Guidelines for assessing sex offenders", dans L.E. Walker (dir.), Handbook of sexual abuse of children. New York: Springer Publishing Co.

MEYER WILLIAMS, L.M. et D. FINKELHOR (1990). "The characteristics of incestuous fathers: A review of recent studies », dans W. Marshall et al. (dir.), Handbook of sexual assault. New York: Plenum.

MoOS, R.H. et B.S. MoOS (1986). Family Environment Scale Manual (Second ed.). Palo Alto, CA: Consulting Psychologists Press.

NASH, M.R., T.L. HulSEY, M.-C. SEXTON, T.L. HARRALSON et W. LAMBERT (1993). «Long term sequelae of childhood sexual abuse: Perceived family environment, psychopathology and dissociation », Journal of Consulting and Clinical Psychology, 61: 276-283.

NiCHOLS, H.R. et I. MOLINDER (1984). Multiphasic Sex Inventory Manual, 437, Bowes Drive, Tacoma, WA 98466.

PARADIS, J.-P., A. Perron et J. Dubé (1992). « Un programme d'évaluation et de traitement des abus sexuels intrafamiliaux», PRISME, vol. 3, n ${ }^{\circ} 1$ : 123-132.

PAulhus, D.L. (1984). "Two component models of socially desirable responding ", Journal of Personality and Social Psychology, vol. 46 : 598609.

RIBORDY, S.C. (1989). "Treating intrafamilial child sexual abuse from a systemic perspective ", Journal of Psychotherapy and the Family, vol. 6, n 3-4 : 71-88.

SAbOurin, S., N. LAferrière, F. SiCuro, J.-C. COAllier, L.-G. COURnOyer et P. GENDREAU (1989). "Social desirability, psychological distress and client satisfaction with mental health treatment», Journal of Counselling Psychology, 36: 351-356.

SABOURIN, S., J. WRIGHT, Y. LUSSIER et C. BÉLANGER (1993). Les recherches descriptives sur les enfants victimes d'abus sexuel et leur famille: recensement et critique. Québec: Université Laval.

SIRLES, E.A. et C.E. LOFBERG (1990). "Factors associated with divorce in intrafamily sexual abuse», Child Abuse \& Neglect, vol. 14: 165-170.

TOURIGNY, M. (1991). "Rapport concernant la littérature scientifique sur I'incidence, les facteurs de risque et les programmes de prévention des abus sexuels envers les enfants ». Étude présentée au Gouvernement du Québec. Un Québec fou de ses enfants. Rapport du Groupe de travail 
pour les jeunes, ministère de la Santé et des Services sociaux (Rapport Bouchard).

TOURIGNy, M., M. PÉlAdeAu et M. Doyon (1993). Évaluation sommative du programme de traitement des enfants abusés sexuellement (PETAS) du Centre des services sociaux Laurentides-Lanaudière dans la région de Lanaudière. Rapport final soumis à Santé et Bien-être Canada.

TOUSIGNANT, M. et V. KOVESS (1985). "L'épidémiologie en santé mentale: Le cadre conceptuel de l'enquête", Santé Québec. Sociologie et Société, vol. 17: 15-19.

TREPPER, T.S. et D.H. SPRENKLE (1988). "The clinical use of the circumplex model in the assessment and treatment of intrafamily child sexual abuse», Journal of Psychotherapy and the Family, vol. 4: 93-111.

VALLERAND, R.J. et E.S. VALLIÈRES (1990). «Traduction et validation canadiennefrançaise de l'échelle de l'estime de soi de Rosenberg "Journal international de psychologie, vol. 25 : 305-316.

VAN GIJSEGHEM, J. et L. GAUTHIER (1992). "De la psychothérapie de I'enfant incestué : les dangers d'un viol psychique », Santé mentale au Québec, vol. XVII, $\mathrm{n}^{\circ} 1: 19-30$.

WAGNER, W.G. (1991). "Depression in mothers of sexually abused vs mothers of nonabused children», Child Abuse \& Neglect, vol. 15:99-104.

WHITMAN, W.P. et V.L. QUINSEY (1981). "Heterosocial skill training for institutionalized rapists and child molesters», Canadian Journal of Behavioral Science, vol. 13:105-114.

WOODWORTH, D.L. (1991). «Evaluation of a multiple-family incest treatment program », dans M.Q. Patton (dir.), Family sexual abuse: Frontline research and evaluation. Newbury Park, CA : Sage Publications, p. 121-134.

Wright, J., J.-P. PARAdis, P. Pinard, A. Perron et J.-G. Gagnon (1995). «Étude sur la clientèle et les pratiques en matière d'abus sexuel effectuée au CPEJ de la Maurice-Bois-Francs », Intervention (Soumis pour publication).

Wright, J., S. SABOURIN, T. LEBEAU et al. (1994). Rapport d'étapes du fonds de programmation d'équipe CQRS du PRIMASE. Université de Montréal.

WRIGHT, J., S. SABOURIN, Y. LUSSIER et C. BÉLANGER (1995). Les recherches évaluatives sur les interventions auprès des victimes d'abus sexuel et leur famille: résumé et critique. Université de Montréal.

Wright, J., S. SAbourin, M.-J. Turcotte, P. MCDuff et C. FiChten (1993). La validation d'une mesure de satisfaction sexuelle sur trois échantillons franco-québécois. Département de psychologie, Université de Montréal. 